\title{
NECESSARY AND SUFFICIENT CONDITIONS FOR OSCILLATION OF SECOND ORDER NEUTRAL DIFFERENCE EQUATIONS
}

\author{
E. THANDAPANI AND K. MAHALINGAM
}

Abstract. Consider the second order difference equation of the form

$$
\Delta^{2}\left(y_{n-1}-p y_{n-1-k}\right)+q_{n} f\left(y_{n-\ell}\right)=0, \quad n=1,2,3, \ldots
$$

where $\left\{q_{n}\right\}$ is a nonnegative real sequence, $f: \mathbb{R} \rightarrow \mathbb{R}$ is continuous such that $u f(u)>0$ for $u \neq 0,0 \leq p<1, k$ and $\ell$ are positive integers. We establish the necessary and/or sufficient conditions for the oscillation of all solutions of (E) when $\int$ is linear, superlinear or sublinear and the results reduce to the well known theorems of Hooker and Patula in the special case when $f(u)=u^{\gamma}$, where $\gamma$ is a odd positive integers.

\section{Introduction}

Consider the second order neutral difference equation of the form

$$
\Delta^{2}\left(y_{n-1}-p y_{n-1-k}\right)+q_{n} f\left(y_{n-\ell}\right)=0, \quad n=1,2,3, \ldots
$$

subject to the conditions:

(c $\left.c_{1}\right)\left\{q_{n}\right\}$ is a sequence of real numbers such that $q_{n} \geq 0$ for all $n \geq 1$ and not identically equal to zero for many values of $n$;

$\left(\mathrm{c}_{2}\right) \quad f: \mathbb{R} \rightarrow \mathbb{R}$ is continuous and nondecreasing such that $u f(u)>0$ for $u \neq 0$;

$\left(\mathrm{c}_{3}\right) \quad 0 \leq p<1, k$ and $\ell$ are positive integers.

For any real sequence $\left\{\phi_{n}\right\}$ defined in $-\theta \leq n \leq 0$ where $\theta=\max \{k, \ell\}$, equation (E) has a solution $\left\{y_{n}\right\}$ defined for $n \geq 1$ and satisfying the initial condition $y_{n}=\phi_{n}$ for $-\theta \leq n \leq 0$. A solution $\left\{y_{n}\right\}$ of equation (E) is oscillatory if it is neither eventually positive nor eventually negative and nonoscillatory otherwise.

We shall consider a class of nonlinear function $f$ satisfying certain nonlinear conditions typified by the Emden-Fowler difference equation

$$
\Delta^{2} y_{n-1}+q_{n} y_{n}^{\gamma}=0
$$

where $\gamma$ is a odd positive integers. We say that $f$ satisfies the superlinear condition if

$$
0<\int_{c}^{\infty} \frac{d u}{f(u)} ; \quad \int_{-\infty}^{-c} \frac{d u}{f(u)}<\infty \text { for all } c>0
$$

Received March 1, 2002.

2000 Mathematics Subject Classification. 39A12.

Key words and phrases. Neutral difference equation, second order, oscillation. 
and satisfies sublinear condition if

$$
0<\int_{0}^{c} \frac{d u}{f(u)} ; \quad \int_{-c}^{0} \frac{d u}{f(u)}<\infty \quad \text { for all } c>0 .
$$

Conditions (1) and (2) correspond to $\gamma>1$ and $0<\gamma<1$ in equation $\left(\mathrm{E}_{1}\right)$ respectively.

For the equation $\left(\mathrm{E}_{1}\right)$, there is a necessary and sufficient condition for the oscillation for all its solutions due to Hooker and Patula [10].

Theorem A. Let $q_{n} \geq 0$ for all $n \geq 1$ and not identically zero for many values of $n$. Then, if $\gamma>1$, all solutions of $\left(\mathrm{E}_{1}\right)$ are oscillatory if and only if

$$
\sum_{n=1}^{\infty} n q_{n}=\infty
$$

Theorem B. Let $q_{n} \geq 0$ for all $n \geq 1$ and not identically zero for many values of $n$. Then, if $0<\gamma<1$, all solutions of equation $\left(\mathrm{E}_{1}\right)$ are oscillatory if and only if

$$
\sum_{n-1}^{\infty} n^{\gamma} q_{n}=\infty
$$

For $f(u)=u$ and $p \equiv 0$, equations (E) reduces to a linear delay difference equation of the form

$$
\Delta^{2} y_{n-1}+q_{n} y_{n-\ell}=0 .
$$

Recently Grzegorezyk and Werbowski [4] estabilished a sufficient condition for the oscillation of all solutions of $\left(\mathrm{E}_{3}\right)$.

Theorem C. Let $q_{n} \geq 0$ for all $n \geq 1$ and not identically zero for many values of $n$. Then every solution of equation $\left(\mathrm{E}_{3}\right)$ is oscillatory if

$$
\lim _{n \rightarrow \infty} \inf \sum_{s=n-\ell}^{n-1}(s-\ell-1) q_{s}>\left(\frac{\ell}{\ell+1}\right)^{\ell+1} .
$$

The purpose of this paper is to prove analogous results of Theorems A, B and C for the neutral difference equation (E). As a general reference on oscillation theory for neutral difference equations, we refer to the recent monographs by Agarwal [1] and Agarwal and Wong [4]. Oscillation theory for second order neutral difference equations were discussed by Thandapani etal. [2, 3, 13], Szafranski and Szmanda [11], Budincevic [5], Grace and Lalli [6], Zafar and Dahiya [15] and Zhon and Zhang [16]. In the delay difference case, that is, equation (E) with $p \equiv 0$, reference should also be made to Györi and Ladas [8]. 
Extension of Hooker and Patula oscillation theorems to more general nonlinear difference equations were given in $[9,12,14]$.

\section{Some Preliminary Lemmas}

In this section we state and prove some lemmas which are useful in establishing the main results of this paper.

Lemma 1. Let $\left\{y_{n}\right\}$ be an eventually positive solution of equaiton (E) and define

$$
z_{n}=y_{n}-p y_{n-k}
$$

Then there is a positive integer $N \geq 1$ such that $z_{n}>0$ and $\Delta z_{n}>0$ for all $n \geq N$.

Proof. Since $\left\{y_{n}\right\}$ is an eventually positive solution of equation (E). We may assume that $y_{n}>0, y_{n-k}>0$ and $y_{n-\ell}>0$ for all $n \geq N_{1}$ for some positive integer $N_{1}$ depending on the solution $\left\{y_{n}\right\}$. Since $y f(y)>0$ and $q_{n} \geq 0$, for $n \geq N_{1}$ equation (E) implies that $\Delta^{2} z_{n-1} \leq 0$ and $\Delta z_{n-1}$ is nonincreasing. Hence $\lim _{n \rightarrow \infty} \Delta z_{n-1}=c$. Suppose $c<0$. Then clearly $\lim _{n \rightarrow \infty} z_{n}=-\infty$. We claim that $\left\{z_{n}\right\}$ cannot be eventually negative for $n \geq N_{1}$. Suppose it is the case, consider two mutually exclusive cases:

(a) there exists a sequence of positive integers $\left\{s_{j}\right\}$ such that $s_{j} \rightarrow \infty$ as $j \rightarrow \infty$ and $y_{s_{j}}=\sup _{n<s_{j}} y_{n}$ or otherwise,

(b) there exists a sequence $\left\{m_{j}\right\}$ of positive integers such that $m_{j} \rightarrow \infty$ as $j \rightarrow \infty$ and $y_{m_{j}}=\inf _{n \leq m_{j}} y_{n}$.

In the first case (a), we have

$$
z_{s_{j}}=y_{s_{j}}-p y_{s_{j-k}} \geq y_{s_{j}}(1-p)>0
$$

which shows that $\left\{z_{n}\right\}$ cannot be eventually negative. In the case (b), we have

$$
z_{m_{j+k}}=y_{m_{j+k}}-p y_{m_{j}} \geq y_{m_{j}}(1-p)>0
$$

which again shows that $\left\{z_{n}\right\}$ cannot be eventually negative. In particular $c<0$ is not possible. Thus we must have $c \geq 0$ which implies that $\left\{z_{n}\right\}$ must be eventually positive, that is, there exists a positive integer $N \geq N_{1}$ such that $z_{n}>0$ for all $n \geq N$. Otherwise, since $\lim _{n \rightarrow \infty} \Delta z_{n}=c \geq 0$ and $\left\{\Delta z_{n}\right\}$ is nonincreasing, we must have $\Delta z_{n}<0$ for all sufficiently large $n$. Then there exists a positive integer $N_{2}>N$ such that $\Delta z_{n}<\Delta z_{N_{2}}<$ 0 and we find that $\left\{z_{n}\right\}$ is eventually negative. We therefore have $z_{n}>0$, and $\Delta z_{n}>0$ for all $n \geq N$. This completes the proof of the lemma.

Lemma 2. Let $z_{n}>0, \Delta z_{n}>0$ and $\Delta^{2} z_{n} \leq 0$ for all $n \geq N \geq 1$. Then $z_{n} \geq$ $(n-1) \Delta z_{n-1}$ for all $n \geq N$.

Proof. From the equation

$$
z_{n}=z_{N}+\sum_{s=N}^{n-1} \Delta z_{s}
$$


we obtain, in view of the nonincreasing nature of $\left\{\Delta z_{n}\right\}$, that

$$
z_{n} \geq z_{N}+(n-N) \Delta z_{n-1} \geq(n-1) \Delta z_{n-1}
$$

for all $n \geq N$. This completes the proof of the lemma.

\section{Main Results}

In this section, first we shall establish a necessary and sufficient condition for the oscillation of all solutions of equation (E) if the nonlinear function $f$ satisfied the superlinear condition (1) and a Lipshitz condition on the given interval; that is there is a number $L$ such that

$$
|f(x)-f(y)| \leq L|x-y| \quad \text { for all } \quad x, y \in\left[\frac{1}{2}, \frac{1}{1-p}\right]
$$

Theorem 3. With respect to the difference equation (E), suppose that conditions (1) and $(7)$ hold. Then all solutions of equation (E) are oscillatory if and only if condition (3) holds.

Proof. To prove sufficiency, let $\left\{y_{n}\right\}$ be a nonoscillatory solution of equation (E). Since $y f(y)>0$ whenever $y \neq 0$, we may without loss of generality assume that $y_{n}>0$ for all $n \geq n_{0} \geq 1$ for some positive integer $n_{0}$, depends on the solution $\left\{y_{n}\right\}$. Then from Lemma 1 , there is a positive integer $N_{1} \geq n_{0}$ such that

$$
z_{n}>0, \quad \Delta z_{n}>0 \text { and } \Delta^{2} z_{n-1} \leq 0
$$

for all $n \geq N_{1}$. Since $f$ is nondecreasing and therefore from equation (E) and (6), we have

$$
\Delta^{2} z_{n-1}+q_{n} f\left(z_{n-\ell}\right) \leq 0
$$

for all $n \geq N \geq N_{1}+\ell$. Define

$$
W_{n}=\frac{n \Delta z_{n-1}}{f\left(z_{n-\ell}\right)}, \quad n \geq N .
$$

then, inview of (8), $W_{n}>0$ for $n \geq N$ and satisfies on account of (9), the Riccati difference inequality,

$$
\Delta W_{n}+n q_{n} \leq \frac{\Delta z_{n}}{f\left(z_{n-\ell}\right)}-\frac{(n+1) \Delta z_{n} \Delta f\left(z_{n-\ell}\right)}{f\left(z_{n-\ell}\right) f\left(z_{n+1-\ell}\right)} .
$$

Inview of condition $\left(\mathrm{c}_{2}\right)$ and from the nature of $\left\{\Delta z_{n}\right\}$, we have from (10),

$$
\Delta W_{n}+n q_{n} \leq \frac{\Delta z_{n-\ell-1}}{f\left(z_{n-\ell}\right)}, \quad n \geq N
$$


Summing the last inequality from $N$ to $n$, we obtain

$$
W_{n}+\sum_{s=N}^{n} s q_{s} \leq W_{N}+\sum_{s=N}^{n} \frac{\Delta z_{s-\ell-1}}{f\left(z_{s-\ell}\right)}
$$

Let $r(t)=z_{n-1-\ell}+\Delta z_{n-1-\ell}(t-n), n \leq t \leq n+1$. Then $r(n)=z_{n-1-\ell}, r(n+1)=z_{n-\ell}$ and $r^{\prime}(t)=\Delta z_{n-1-\ell}, n<t<n+1$. Thus $r(t)$ is continuous and increasing for $t \geq N$. We then have

$$
\frac{\Delta z_{s-\ell-1}}{f\left(z_{s-\ell}\right)}=\int_{s}^{s+1} \frac{\Delta z_{s-\ell-1}}{f\left(z_{s-\ell}\right)} d t=\int_{s}^{s+1} \frac{r^{\prime}(t)}{f\left(z_{s-\ell}\right)} d t<\int_{s}^{s+1} \frac{r^{\prime}(t)}{f(r(t))} d t .
$$

This implies that

$$
\sum_{s=N}^{n} \frac{\Delta z_{s-\ell-1}}{f\left(z_{s-\ell}\right)} \leq \int_{z_{N-1-\ell}}^{z_{n-\ell}} \frac{d u}{f(u)}
$$

From (11) and (12) we obtain

$$
\sum_{s=N}^{n} s q_{s} \leq W_{N}+\int_{z_{N-\ell-1}}^{\infty} \frac{d u}{f(u)}-\int_{z_{n-\ell-1}}^{\infty} \frac{d u}{f(u)} \leq M_{0}
$$

where $M_{0}$ depends only on the solution $\left\{y_{n}\right\}$. Letting $n \rightarrow \infty$ in (13) one easily sees that it is incompatible with the condition (3). This proves the sufficient part of the theorem.

To prove the necessity of condition (3) for the oscillation of all solution of the equation (E), we shall apply the contraction mapping principle. Consider the Banach space $\mathcal{B}_{N}$ of all bounded real sequences $\left\{y_{n}\right\}, n \geq N$ with the norm defined as $\|y\|=\sup _{n \geq N \geq 1}\left|y_{n}\right|$ where the positive integer $N$ to be chosen later. Assume that the condition (3) fails; that is $\sum_{n-1}^{\infty} n q_{n}<\infty$, then there is a nonoscillatory solution $\left\{y_{n}\right\}$ for the equation $(\mathrm{E})$. We shall show the existence of a solution $\left\{y_{n}\right\}$ of equation (E) such that $\lim _{n \rightarrow \infty} y_{n}=\frac{1}{1-p}$. Let $\mathcal{S}$ be a closed bounded subset of $\mathcal{B}_{N}$ such that

$$
\mathcal{S}=\left\{y \in \mathcal{B}_{N}: \frac{1}{2} \leq y_{n} \leq \frac{1}{1-p}, n \geq N\right\}
$$

Define the operator $\mathcal{T}: \mathcal{S} \rightarrow \mathcal{B}_{N}$ such that

$$
\mathcal{T} y_{n}=1+p y_{n-1-k}-\sum_{s=n+1}^{\infty}(s-n) q_{s} f\left(y_{s-\ell}\right)
$$

Choose a positive integer $N$ sufficiently large so that $L \sum_{n=N}^{\infty} n q_{n} \leq \frac{1-p}{2}$. Let $y \in \mathcal{S}$, then from (15) we have

$$
\mathcal{T} y_{n} \geq 1+\frac{p}{2}-\frac{L}{1-p} \sum_{s=n+1}^{\infty} s q_{s}=1+\frac{p}{2}-\frac{1}{2} \geq \frac{1}{2}
$$


and

$$
\mathcal{T} y_{n} \leq 1+\frac{p}{1-p}=\frac{1}{1-p}
$$

So $\mathcal{T S} \subseteq \mathcal{S}$. On the other hand, using (7) in (15), we find for $x, y \in \mathcal{S}$,

$$
\begin{aligned}
\left|\mathcal{T} y_{n}-\mathcal{T} x_{n}\right| & \leq p\left|y_{n-1-k}-x_{n-1-k}\right|+L \sum_{s=n+1}^{\infty}(s-n) q_{s}\left|y_{s-\ell}-x_{s-\ell}\right| \\
& \leq\left(p+\frac{1-p}{2}\right)\|y-x\| .
\end{aligned}
$$

Therefore, $\|\mathcal{T} y-\mathcal{T} x\| \leq\left(\frac{1+p}{2}\right)\|y-x\|$, and hence $\mathcal{T}$ is a contraction on $\mathcal{S}$. Thus, $\mathcal{T}$ has a unique fixed point in $\mathcal{S}$, which is our desired nonoscillatory solution of (E) such that $\lim _{n \rightarrow \infty} y_{n}=\frac{1}{1-p}$. This completes the proof.

Next we shall prove an analogous result for the oscillation of all solutions of equation (E) in the sublinear case.

Theorem 4. In addition to the condition (2) assume that

$$
f(u v) \geq f(u) f(v) \quad \text { if } \quad u v>0 \quad \text { and } \quad|v| \geq M
$$

for large $M>0$. Then all solutions of the equation (E) are oscillatory if and only if

$$
\sum_{n=1}^{\infty} f(n) q_{n}=\infty
$$

Proof. Let $\left\{y_{n}\right\}$ be a nonoscillatory solution of the equation (E) which can be assumed to be positive for $n \geq N_{1}$ for some positive integer $N_{1}$ and proceed as in the proof of Theorem 3, we obtain

$$
\Delta^{2} z_{n-1}+q_{n} f\left(z_{n-\ell}\right) \leq 0, \quad n \geq N \geq N_{1}+\ell .
$$

From Lemma 2 , we have $z_{n-\ell} \geq(n-\ell-1) \Delta z_{n-1}$ and so $f\left(z_{n-\ell}\right) \geq f\left((n-\ell-1) \Delta z_{n-1}\right)$. For any $\lambda, 0<\lambda<1$ if $N$ is sufficiently large then $(n-\ell-1) \geq \lambda n$ for $n \geq N$. Thus, by (16) we have

$$
f\left((n-\ell-1) \Delta z_{n-1}\right) \geq f\left(\lambda n \Delta z_{n-1}\right) \geq f(n) f\left(\lambda \Delta z_{n-1}\right)
$$

for $n \geq N_{1}$, from which (18) can be rewritten as follows

$$
\frac{\Delta^{2} z_{n-1}}{f\left(\lambda \Delta z_{n-1}\right)}+f(n) q_{n} \leq 0, \quad n \geq N .
$$

For $\lambda \Delta z_{n} \leq t \leq \lambda \Delta z_{n-1}$ we have $f(t) \leq f\left(\lambda \Delta z_{n-1}\right)$ and so

$$
\lambda f(n) q_{n} \leq-\int_{\lambda \Delta z_{n-1}}^{\lambda \Delta z_{n}} \frac{d t}{f(t)}
$$


for $n \geq N$. Summing the last inequality from $N$ to $n$, we obtain

$$
\lambda \sum_{s=N}^{n} f(s) q_{s} \leq \int_{\lambda \Delta z_{n}}^{\lambda \Delta z_{N-1}} \frac{d t}{f(t)}<\int_{0}^{\lambda \Delta z_{N-1}} \frac{d t}{f(t)}<\infty
$$

which is incompatible with the condition (17). This proves the sufficiency part of the theorem.

To prove that condition (17) is also necessary for the oscillation of all solutions of equation (E), we assume that the condition (17) fails and proceed to establish the existence of a nonoscillatory solution. In this case we choose $N$ sufficiently large such that $\sum_{n=N}^{\infty} q_{n} f(n)<\lambda \frac{(1-p)}{4}$, where $0<\lambda<1$. Let $\theta=\max (k, \ell)>0$ and $\mathbb{N}(\theta, N)=$ $\{N-\theta, N-\theta+1, \ldots, N\}$. Consider the sequence $\left\{\phi_{n}\right\}$ defined by $\phi_{s}=\lambda(s-N+\theta)$ for $s \in \mathbb{N}(\theta, N)$. Here $\phi_{s} \geq 0, \Delta \phi_{s}=\lambda$ for all $s \in \mathbb{N}(\theta, N), \phi_{N}=\lambda \theta>0$ and $\phi_{N-k}=\lambda(\theta-k) \geq 0$. For such a given initial sequence $\left\{\phi_{n}\right\}$, the difference equation (E) has a solution $\left\{y_{n}\left(\phi_{n}\right)\right\}$ which we shall denote by $\left\{y_{n}\right\}$ for short and $y_{n}=\phi_{n}$ for all $n \in \mathbb{N}(\theta, N)$. We shall prove that this solution is nonoscillatory. In fact $\Delta y_{N}=\Delta \phi_{N}=\lambda$ and we shall show that $\Delta y_{n} \geq \frac{\lambda}{2}$ for all $n \geq N \in \mathbb{N}(N, j-1)=\{N, N+1, \ldots, j-1\}$. Then $y_{n}>0$ for all $n \in \mathbb{N}(N, j)$. However from the equation (E), $\Delta^{2} z_{n-1} \leq 0$ for all $n \in \mathbb{N}(N, j)$ and therefore from (6) we find $\Delta^{2} y_{n-1} \leq 0$ for all $n \in \mathbb{N}(N, j)$. Then, for all $n \in \mathbb{N}(N+1, j)$, it follows that

$$
y_{n-\ell} \leq y_{N}+(n-\ell-1) \Delta y_{N} \leq n
$$

Now from equation (E) and the above inequality, we obtain

$$
\begin{aligned}
\Delta z_{j} & =\Delta z_{N}-\sum_{s=N+1}^{j} q_{s} f\left(y_{s-\ell}\right) \\
& \geq \lambda(1-p)-\sum_{s=N+1}^{j} q_{s} f(s) \\
& \geq \lambda(1-p)-\frac{\lambda(1-p)}{2} \\
& =\frac{\lambda(1-p)}{2} .
\end{aligned}
$$

Since $\Delta z_{j+k}=\Delta y_{j+k}-p \Delta y_{j}$ and $\Delta y_{j}$ is nonincreasing we have $(1-p) \Delta y_{j} \geq \frac{\lambda}{2}(1-p)$ and therefore $\Delta y_{j} \geq \frac{\lambda}{2}$. Now by induction $\Delta y_{n} \geq \frac{\lambda}{2}$ for all $n \in \mathbb{N}$. This completes the proof.

Finally we shall prove an analogous result for the oscillation of all solutions of equation (E) in the linear case, that is,

$$
\frac{f(u)}{u} \geq M>0 \quad \text { for } u \neq 0 .
$$


Theorem 5. In addition to the condition (19) assume that

$$
\lim _{n \rightarrow \infty} \inf \sum_{s=n-\ell}^{n-1}(s-\ell-1) q_{s}>\frac{1}{M}\left(\frac{\ell}{\ell+1}\right)^{\ell+1} .
$$

Then every solution of the equation (E) is oscillatory.

Proof. Let $\left\{y_{n}\right\}$ be a nonoscillatory solution of the equation (E) which can be assumed to be positive for $n \geq N_{1}$ for some positive integer $N_{1}$ and proceed as in the proof of Theorem 3, we obtain the inequality (18). Using condition (19) in (18). We have

$$
\Delta^{2} z_{n-1}+M q_{n} z_{n-\ell} \leq 0, \quad n \geq N
$$

From Lemma 2, we have

$$
z_{n-\ell} \geq(n-\ell-1) \Delta z_{n-\ell-1}, \quad n \geq N .
$$

Combining (21) and (22), we obtain

$$
\Delta^{2} z_{n-1}+M(n-\ell-1) q_{n} \Delta z_{n-\ell-1} \leq 0 .
$$

Let $x_{n}-\Delta z_{n-1}$. Then $\left\{x_{n}\right\}$ is eventually positive and from (23), satisfies the inequality

$$
\Delta x_{n}+M(n-\ell-1) q_{n} x_{n-\ell} \leq 0, \quad n \geq N .
$$

In view of condition (20), inequality (24) has no positive solutions, a contradiction. This completes the proof.

Remark 1. The results in this paper are presented in a form which is essentially new. The results obtained in this paper improves some of the results obtained in $[2,3$, $13,15]$.

\section{References}

[1] R. P. Agrwal, Difference Equations and Inequalities, Second Edition Marcel Dekker, New york, 2000 .

[2] R. P. Agarwal, M. M. S. Manucl and E. Thandapani, Oscillatory and nonoscillatory behavior of second order neutral delay difference equations, Mathl. Comput. Modelling 24(1996), $5-11$.

[3] R. P. Agarwal, M. M. S. Manuel and E. Thandapani, Oscillatory and nonoscillatory behavior of second order neutral delay difference equations, II Appl. Math. Letters 10(1997), 103109.

[4] R. P. Agarwal and P. J. Y. Wong, Advanced Topics in Difference Equations, Kluwar Pub. Dordrecht, 1997.

[5] M. Budincevic, Oscillation of a second order neutral difference equation, Bull. Cl. Sci. Math. Nat. Sci. Math. 22(1994), 1-8. 
[6] S. R. Grace and B. S. Lalli, Oscillation theorems for second order delay and neutral difference equations, Utilitas Math. 45(1994), 197-211.

[7] G. Grzegorczyk and J. Werbowski, Oscillation of higher order linear difference equation, Comput. Math. Applic. 42(2001), 711-718.

[8] I. Györi and G. Ladas, Oscillation Theory of Delay Differential Equations, Clarender Press, Oxford, 1991.

[9] X. Z. He, Oscillatory and asymptotic behavior of second order nonlinear difference equations, J. Math. Anal. Appl. 175(1993), 482-498.

[10] J. W. Hooker and W. T. Patula, A second order nonlinear difference equation: Oscillation ans asymptotic behavior, J. Math. Anal. Appl. 91(1983), 9-29.

[11] Z. Szafranski and B. Szmanda, Oscillation and asymptotic behavior of certain nonlinear difference equations, Riv. Mat. Univ. Parma 4(1995), 231-240.

[12] E. Thandapani, I. Györi and B. S. Lalli, An application of discrete inequality to second order nonlinear oscillation, J. Math. Anal. Appl. 186(1994), 200-208.

[13] E. Thandapani, P. Sundaram, J. R. Graef and P. W. Spikes, Asymptotic properties of solutions of nonlinear second order neutral delay difference equations, Dyanamic Sys. Appl. 4(1995), 125-136.

[14] P. J. Y. Wong and R. P. Agarwal, Summation averages and the oscillation of second order nonlinear difference equations, Mathl. Comput. Modelling 24(1996), 21-35.

[15] A. Zafer and R. S. Dahiya, Oscillation of a neutral delay difference equations, Appl. Math. Letters 6(1993), 71-74.

[16] Z. Zhon and Q. Zhang, Linearized oscillations for even order netural difference equations, Math. Sci. Res. Hot Line 2(1998), 11-17.

Department of Mathematics, Peryiar University, Salem-636011, Tamilnadu, India. 